

\section{Analysis of Laser Fusion Targets \\ Using Monochromatic X-Ray Microradiographs}

R. L. Whitman
R. H. Day
R. P. Kruger
D. M. Stupin
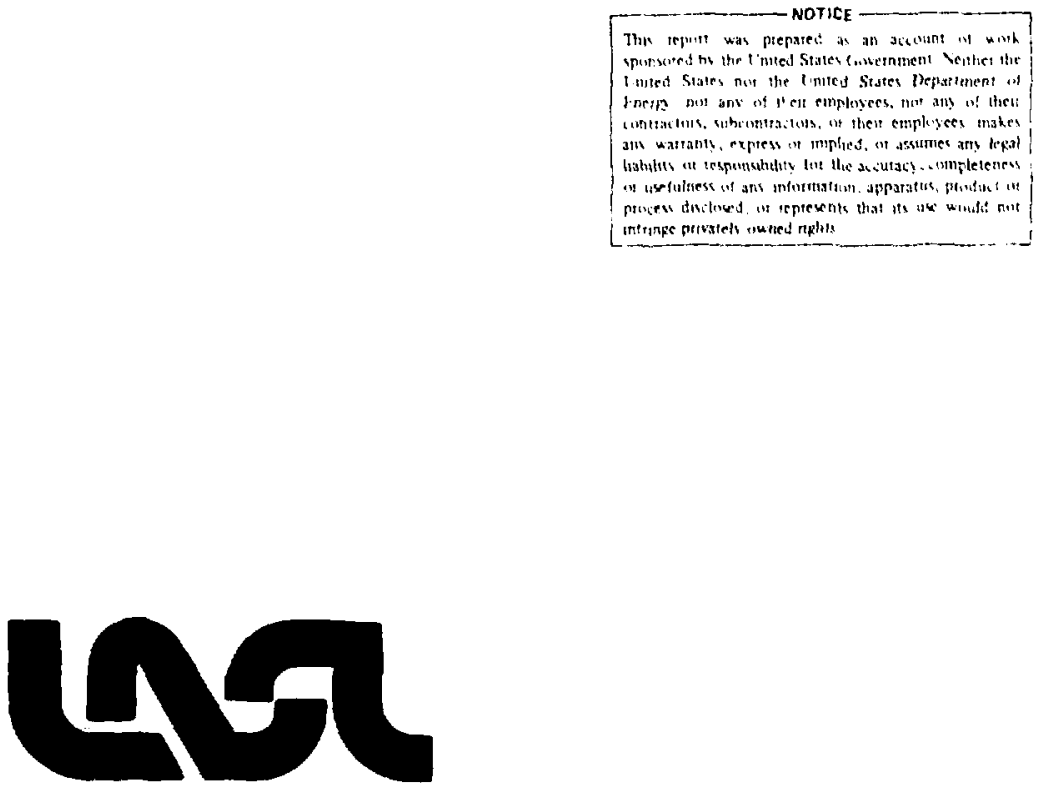
ANALYSIS OF LASER FUSION TARGETS

USING MONOCHROMATIC X-RAY MICRORADIOGRAPHS

by

R. L. Whitman, R. H. Day, R. P. Kruger, and D. M. Stupin

\begin{abstract}
A new contact microradiographic system for analyzing laser fusion targets with two-dimensional modeling and imageanalysis techniqucs is described. This system, which uses a monochromatic $x$-ray source and Kodak High-Resolution Plate emulsion, is sensitive to spherical wall-thickness variations (eccentricities) as small as $\pm 200 \AA$ in hollow shells with a mean wall thickness of $1 \mu \mathrm{m}$. Measurements of wall thickness and of local and spherical wall-thickness variations by radiographic techniques, using two-dimensional video, digjtal image analysis, and optical interferometry, are compared. In addition, three digitizing systems are compared for converting the radiographic data to digital form.
\end{abstract}

I. INTRODUCTION

Theoretical calculations of laser-driven implosions predict that uniform, symmetric shells are required for successful laser fusion targets. To date, the most common target contains the fusible gas within a thin-walled spherical glass shell.* The usable shells are typically $100 \mu \mathrm{m}$ in diameter with 1-um-thick walls and have wall-thickness variations no larger than $\pm 300 \AA$. Optical interferometric techniques are adequate to measure wall-thickness uniformity to $\pm 500 \mathrm{~A}$ and possibly to $\pm 300 \AA .^{1,2}$ However, target designs are becoming more complicated, some of them requiring optically opaque, multilayered materials. Therefore, highly symmetric opaque shells will be required, and methods must be

*Several trademarks have been registered for this generic class of target: KMS Fusion, Inc., Microshell, as well as Emmerson and Cummings, Inc., Glass Microballoon, and Eccosphere. 
developed to characterize them. $x$-ray microradiography has been suggested as an appropriate characterization technique. ${ }^{3}$

We will describe a contact microradiographic system comprising monochromatic $x$-ray radiation, Kodak High-Resolution Plate (HRP) emilsion, a twodimensional analytic model, and digital image analys is techniques to inspect opaque shells used as laser fusion targets. Monochromatic $x$ rays from a modified Henke $x$-ray tube illuminate the hollow shells, and the images of the shells are recorded on HRP emulsion (Section II). These microradiographs are digitized into optical density values, and by a sectoring algorithm the digitized image of the hollow shell is partitioned into many equal sectors. Differences in average optical density of opposing sectors are averaged and converted to eccentricity (sphericity) using the results of computer-generated curves from two-dimensional hollow shell models (Sections III and IV). A statistical outlier test is also performed to detect local defects more than two standard deviations from the average sector densities in the hollow shells (Section IV). For convenience we divide the experimentally observed defects into three classes and demonstrate the sensitivity of our method in detecting each class (Sections IV and V). Its sensitivity will be compared to the sensitivities of optical interferometry measurements and radiographic measurements using analog video analysis techniques (Section VI). In addition, three image digitizing systems are compared for scanning the microradiographs (Section VIi).

\section{RADIOGRAPHIC SYSTEM}

To achieve the highest sensitivity to defects, the three major components of the radiographic system must be optimized. They are (1) the $x$-ray source, (2) the microsphere film-imaging system, and (3) the radiograph readout systeri, each of which is discussed in detail below.

A. $\quad x$-ray Source

Two $x$-ray sources have been used at the Los Alamos Scientific Laboratory (LASL) to radiograph laser fusion targets. The primary source used for exposures with ultrasoft $x$ rays $(<1 \mathrm{keV})$ is a modified Henke $x$-ray tube wi in interchangeable anodes to allow us to excite various ultrasoft $x$-ray lines from 0.109 to $1.5 \mathrm{keV} .{ }^{4}$ This flexibility permits us to choose the $x$-ray energy that will produce the maximum contrast in the film image. ${ }^{5}$ The anode potential is restricted to about twice the characteristic line energy to suppress high-energy bremsstrahlung. By filtering the $x$-ray beam, we can restrict the spectral contaminants to 
less than 10\% of the total fluence. To achieve maximum fluence at low anodecathode voltages, we have replaced the tungsten filament with thorium-oxide (ThO)-coated Ir ribbons placed directly in front of the anode. The high emis sivity of these filaments at low temperatures $\left(1500-2000^{\circ} \mathrm{C}\right)$ keeps the surface contamination to negligible levels, as verified by crystal spectroscopy. We are using the $\mathrm{CuL}_{c}$ line at $930 \mathrm{eV}$ to radiograph 1 - $\mu \mathrm{m}$-thick glass shells, and are developing a high-intensity, ultrasoft $x$-ray source using a modified Pierce electron gun to replace the Henke source.

B. Microsphere Film-Imaging System

The inages are contact microradiographs on Kodak HRP emulsion. A schematic of the geometry used with the monoenergetic source is shown in Fig. 1. Contact radiography has several advantages for our application. First, large-area

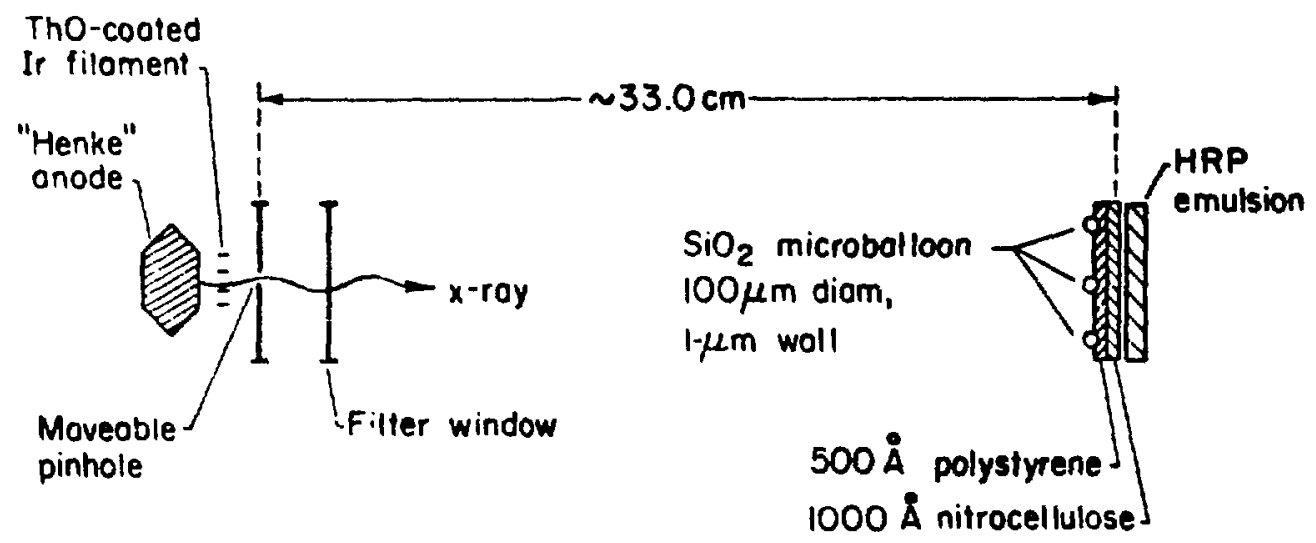

Fig. 1. Geometry of x-ray exposure system for contact microradiography with monoenergetic source.

$x$-ray sources are amenable to broad field illumination, allowing us to expose many microspheres (laser fusion targets) simultaneously. Second, the resolution of the technique is better than that of our ontical readout system. Third, the proximity of the film to the targets makes it easier to construct mechanically rigid mounts to decrease image blurring from vibration.

The resolution limit of the radiographic data is set by four factors: (1) by the geometrical resolution defined by the source size and source-objectfilm geometry, (2) by the resolution of the recording medium, (3) by the 
resolution of the image readout system, and (4) by the resolution due to $x$-ray diffraction in the object. For maximum speed and sensitivity of the system, the first three of these limits of resolution should be comparable in size while ensuring that image blurring due to $x$-ray diffraction is negligible. These three resolution limits are: geometric resolution, $0.5 \mu \mathrm{m}$; resolution of Kodak HRP, $0.5 \mathrm{\mu m}$; and picture-element (pixel)size of the image readout system (a Photometric Data Systems [PDS] Model 1050 Scanning Densitometer), $2.0 \mu \mathrm{m}$. These estimates are higher than the $x$-ray diffraction limit of resolution, $0.06 \mu \mathrm{m}$, for the geometry and monochromatic $x$-ray energy used here. ${ }^{6}$ Therefore, the limit of resolution of the present system is defined by the 2- $-\mu \mathrm{m}$ pixel size of the image-scanning system. In comparison, the best optical microscopes would have been an almost ten times higher resolution and would be used if such fine detail were important for future applications.

object mounting for contact microradiography imposes the additional constraints: (1) the microsphere support structure should be vacuum-compatible and should not absorb the exposing $x$-ray radiation, and (2) the microspheres should be removable for use or reorientation. ${ }^{7}$ A technique that would meet both criteria simultaneously has not yet been identified, although several are being investigated. We are presently using a method in which the microspheres are glued by a $500-\AA$ polystyrene film to a $1000-\AA$ nitrocellulose layer. The transparent backing allows us to radiograph the image and to inspect the microsphere interferometrically in the same orientation.

The sensitivity of the HRP emulsion was measured for the $x$-ray energies of interest, and the resultant optical densities versus exposure levels, i.e., $H-D$ curves, are shown in Fig. 2. The specular density was measured with a PDS scanning microdensitometer.

C.

Image Inspection System

The images were read with the PDS densitometer using a 2-by 2-um aperture stepped in 1-um increments in both the $x$ - and $y$-directions over a raster pattern sufficient to cover the image. The noise in the system is due to both the film grain noise of the emuision and the photomultiplier noise of the densitometer (Fig. 3). These noises combine to generate an effective single-point noise, $\sigma_{D}$. The value of $\sigma_{D}$ was found empirically to be well described by a power law ${ }^{5,8}$ for optical densities above 0.1 .

$$
\sigma_{D}=0.062 D^{0.8} \text {. }
$$




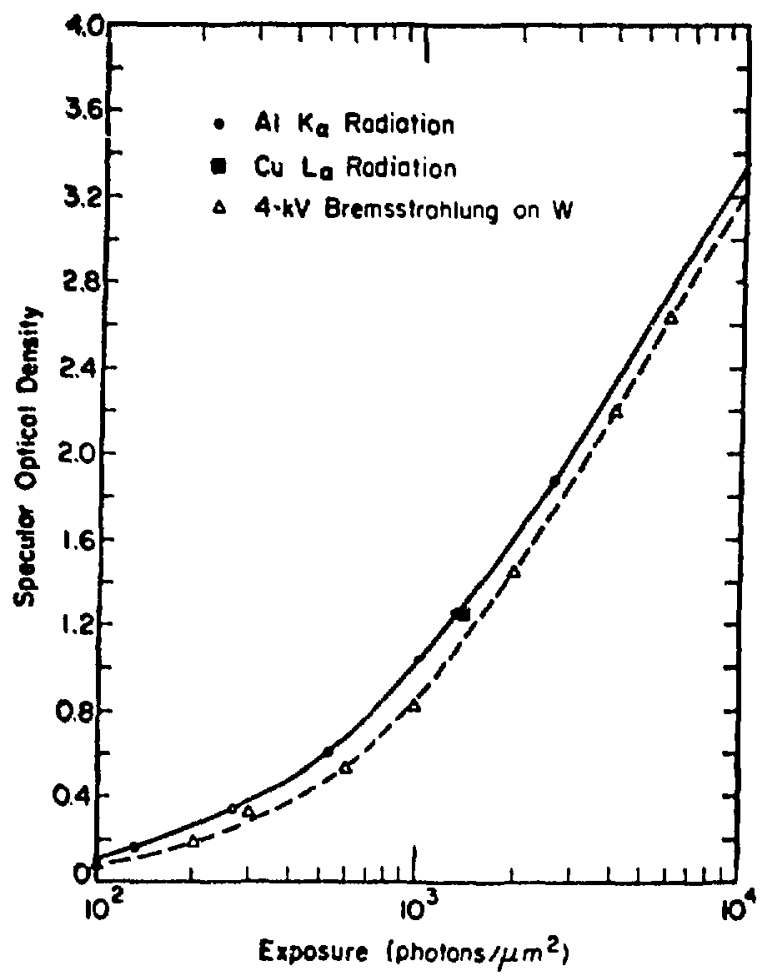

Fig. 2. Total exposure as a function of optical density for HRP emulsion.

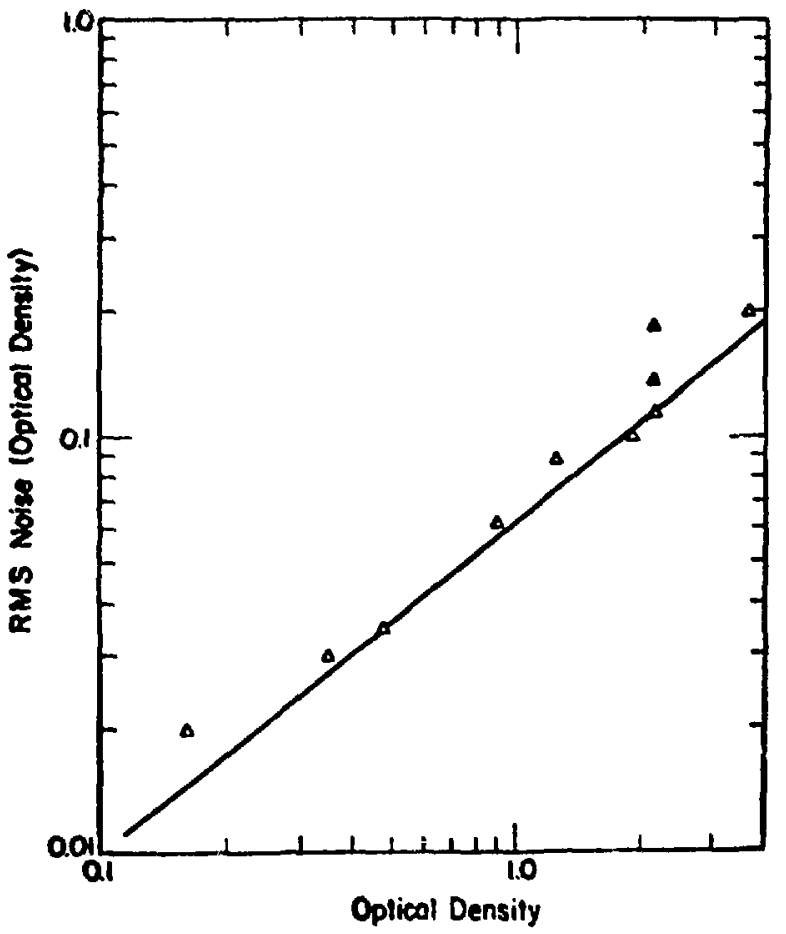

Fig. 3. RMS system noise, $\sigma_{D}$, as a function of HRP emulsion density.

5 
The flatness of the background density is better than 1\% over most of the field, however, there is an additional time-dependent base level shift of about 0.03 optical density units per minute, which necessitates "rezeroing" the densitometer between images. This makes measurements of very low densities less accurate.

\section{TWO-DIMENSIONAL MODEL}

We simulated a two-dimensional $x$-ray radiographic image of an $80-\mu m$-diam glass microsphere with a 1-um-thick wall. This simulation is an extension of a one-dimensional model discussed previous $1 y^{5}$ and is described in Appendix $A$. The simulated images were used as standards to determine the baseline sensitivity of the sphere mensuration algorithm. The model includes as input: the microspluse diameter, wall thickness, wall composition, source size, photon energy, total exposure, the source-to-film distance, microsphere-to-film distarice, the characteristic curve of the film, and the film-recording system noise. Programmed eccentricities of the inner and outer surfaces of the microsphere were introduced to study the sensitivity of the proposed computer eccentricity measurements.

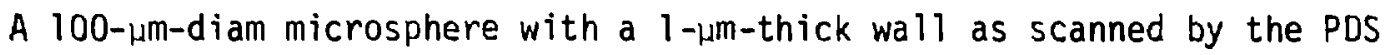
microdensitometer and displayed on a COMTAL digital video is shown in Fig. 4a, and may be compared to a simulated $80-\mu m$-diam microsphere of the same wall thickness, with noise, $\sigma_{D}$, added, as shown in Fig. 4b. Similarly modeled images are used to calibrate our sensitivity to detecting each of three classes of defects, which we discuss in Section IV.

IV. TWO-DIMENSIONAL ANALYSIS FOR THREE CLASSES OF IMAGE DEFECTS

We have experimentally observed three classes of defects in thin-walled microspheres. To analyze their severity, we have devised a two-dimensional analysis technique to detect the three classes of defects. Type-I defects are modeled as an eccentricity of the spherical surfaces defining the inner and outer walls of the microsphere; Type-II defects are modeled as a nonsphericity of one or both of these walls; and Type-III defects are associated with small-scale nonuniformities in the wall composition or thickness (e.g., lumps and holes). We will first outline a general algorithm that is sensitive to detecting Type-I and some Type-II defects; and second we will outline a general algorithm that is sensitive to small Type-II and Type-III defects. 


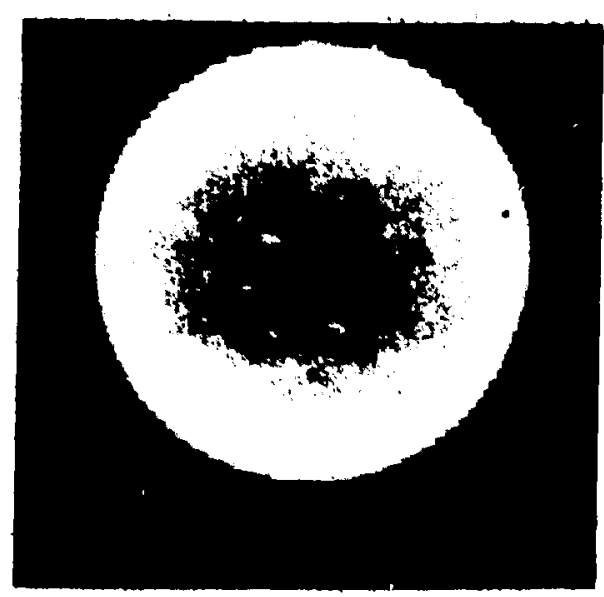

Fig. 4a. Replay of 100-lum-diam microsphere.

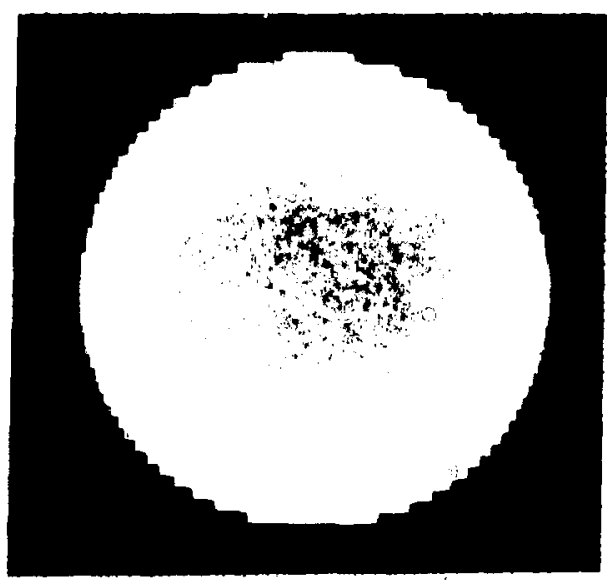

Fig. 4b. Simulated 80-ım-diam microsphere.

\section{A. Type-I Defect Analys is}

For Type-I defects the diametral material thickness of the microsphere is very nearly the same in all views, due to tha decreased path length on the opposite thick side of the microsphere. Therefore, we need a technique for determining the overall symmetry of the image. One general method is to compute the differences in optical density of points displaced symmetrically across the center of the image. The analysis entails five steps: (1) Calculate the radius of the microsphere by using Newton's method ${ }^{9,10}$ to establish a rough center of the microsphere image. (2) Compute a precise center by averaging the $x$ - and $y$-coordinate values of all points whose optical density is below an appropriate threshold value, as shown schematically in Fig. 5. This threshold is the average of the central density, Point 1 in Fig. 5, and of the average optical density in Areas 1, 2, 3, and 4. (3) The data are then translated via bilinear interpolation to a new grid that is symmetric about this computed center. (4) The image is then divided into sectors of angular width $\theta$ and each sector is divided into bins of radial width equal to the sampling aperture as shown in Fig. 6. (5) The average optical density in each sector is calculated and the averaged sum of the absolute value of the density difference of opposing sectors is then used as a measure of the symmetry of the microsphere. This measure of symmetry will be converted to percent eccentricity by curves developed in Section V.A. 


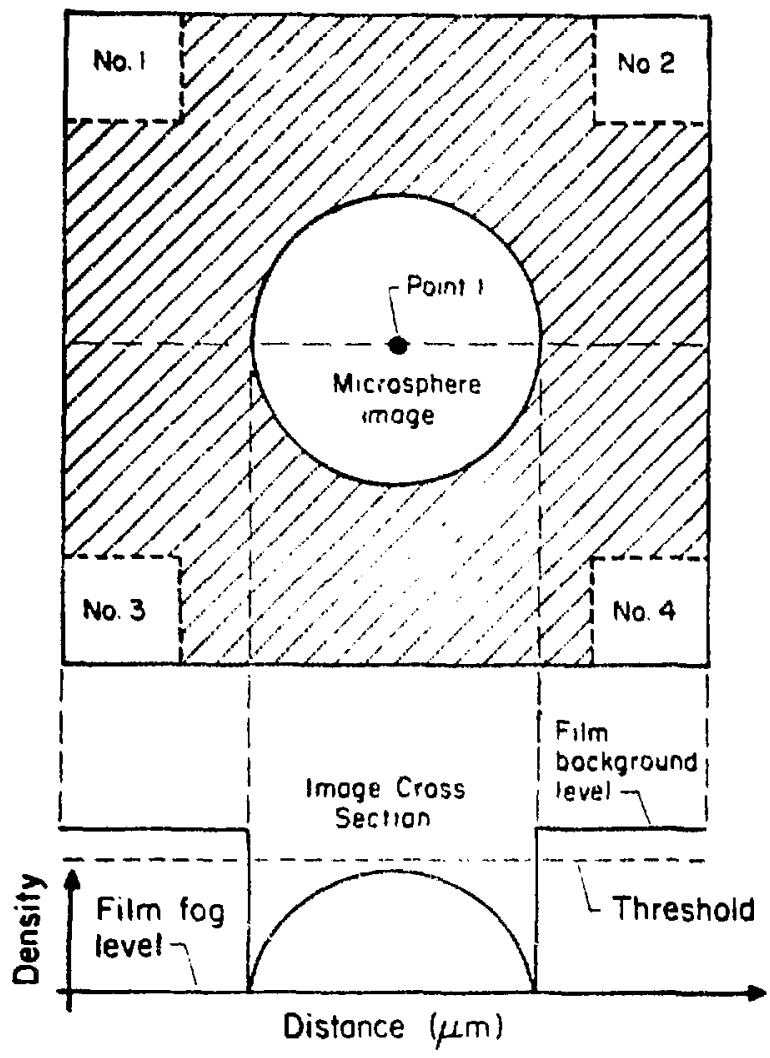

Fig. 5. Diagram of microsphere radiograph and cross section.

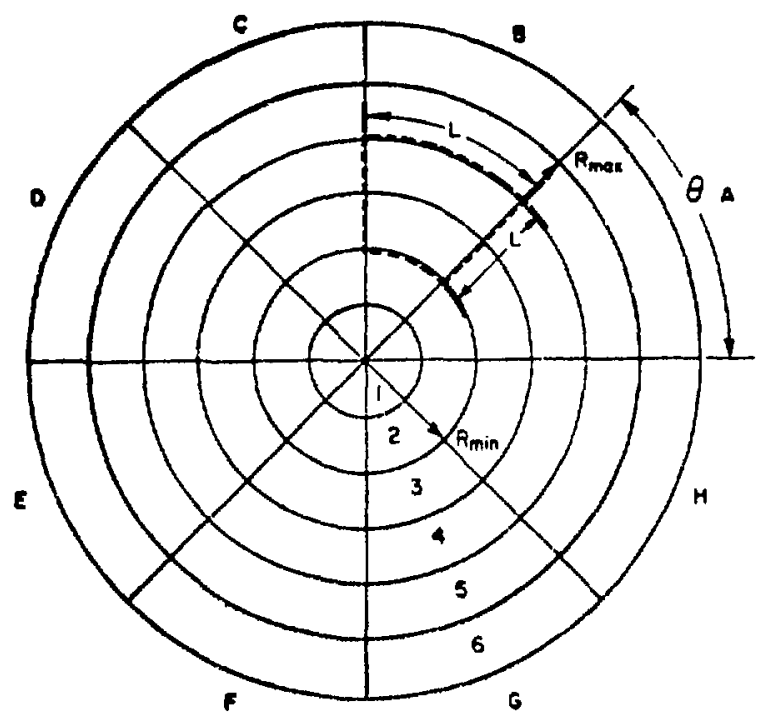

Fig. 6. Sectored microsphere. For maximum sensitivity the calculation considers an annulus from 40 to $80 \%$ of the microsphere radius enclosed by the $R_{\max }$ radii.

\section{B. Type-II and -III Defect Analyses}

For Type-II and Type-III defects we can detect the nonuniformity as a deviation of the observed optical density from the average appropriate for each case. One method for detecting a Type-II defect requires taking at least three identical radiographs of the microsphere in independent orientations and comparing the mean densities in the centers of the images.

For a Type-III defect, we scan the image surface and determine whether statistically significant anomalies occur. We have defined a general technique for doing this, which employs a statistical T-test. 11 This aralysis uses Steps 1 through 4 described above for detecting Type-I defects plus six additional steps: (1) Define an area of approximately $L \times L, m^{2}$ (where $L>1 \mu m$ ) and average the optical densities in that area (Fig. 6). The usual area size is several sector widths by several bin heights (e.g., in Section V.B, $L \approx 7 \mu \mathrm{m}$ ). (2) The $L \times L$ area is stepped around the microsphere at a constant radius by sector increments, resulting in overlapping $L \times L$ adjacent areas, e.g., 2.5 degre ss.

(3) An average 
optical density and uncertainty in the optical density is computed for all overlapping $L \times L$ areas at that radius. (4) The average density is subtracted from all densities for the $L \times L$ areas and divided by the standard deviation in optical density. This results in a signal-to-noise $(S / N)$ ratio $S .(5)$ If this ratio, $S$, is above 2, a "warning" output is given. If above 3 , a "bad" output is given. These limits can be changed. In addition, the location of the defect, the mean wall thickness in micrometers, and the mean defect thickness in micrometers are calculated. (The procedure for calculating the thicknesses is shown in Appendix B.) (6) Steps 2 through 5 are repeated for all $L \times L$ areas at an increased radius of one bin width (usually $1 \mathrm{~mm}$ ). The computation is usually repeated for radii from 10 to $95 \%$ of the computed radius. The output gives a footprint of the small Type-II and Type-III defects.

Taking this technique one step further, it will be nossible to group the overlapping $L \times L$ adjacent defective areas together by a feature-ciustering method to make a composite image of the position, size, shape, and mass of each defect.

V. SENSITIVITY OF TWO-DIMENSIONAL ANALYSIS TECHNIQUES

We next consider the sensitivity of two-dimensional analysis of $x$-ray microradiographs in detecting the types of defects defined earlier. In addition, we list computer resources and execution times based on implementing these twodimensional analysis techniques.

A.

Type-I Defect Sensitivity

The sensitivity for detecting Type-I defects is defined by our ability to detect density differences above the uncertainty (noise) in the measurement. The uncertainty in the measurement arises from both the noise in the film-image readout system and from uncertainity in determining the actual center of the microsphere. Using our two-dimensional analys is technique for Type-I defects, we analyzed the image of a perfect microspherf whose center was intentionally misplaced by $0.1,0.25$, and $0.5 \mu \mathrm{m}$. We also performed this analys is on computer-generated microspheres (generated in accordance with the procedure of Appendix A) with Type-I defects of eccentricity amplitudes of $0.014,0.05,0.1$, and $0.2 \mu \mathrm{m}$. The

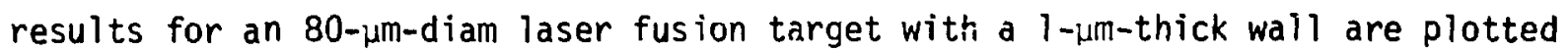
in Fig. $7 .^{12}$ In this figure, signal-to-noise ratio $(S / N)$ is defined as the ratio of the signal (average density asymmetry as defined in Section IV. 8) for the various Type-I defects divided by the 3- $\sigma$ noise. The 3- $\sigma$ noise is calculated by 


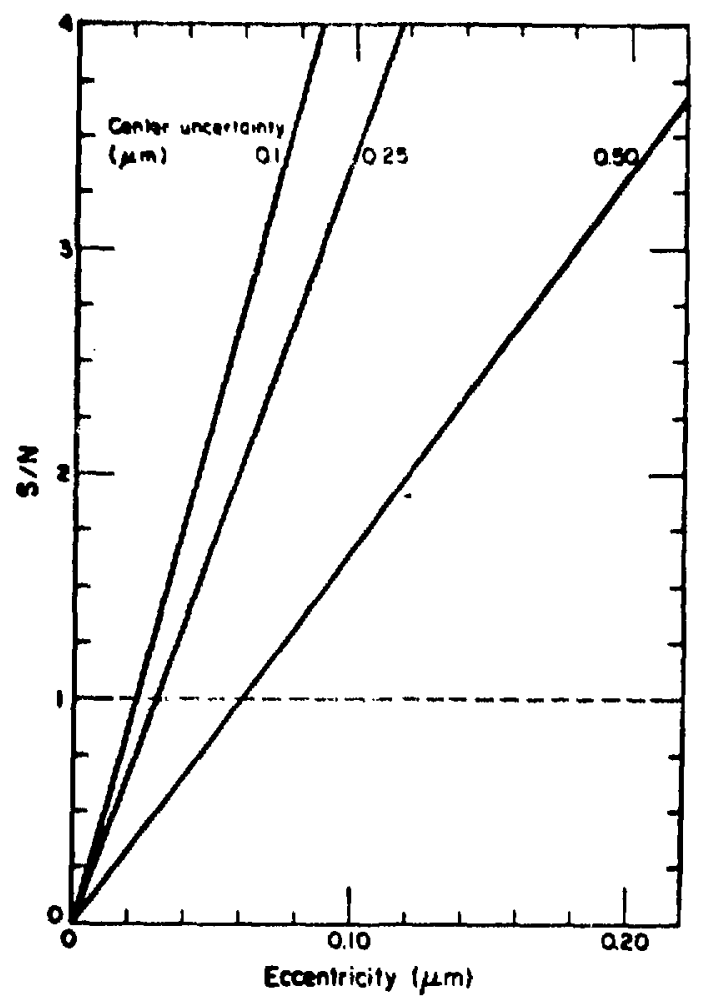

Fig. 7. Sensitivity to defects vs microsphere eccentricity at 930-eV photon energy.

adding the density difference due to uncertainty of the true image center in quadrature with the system noise, $\sigma_{D}$.

For microspheres larger than $40 \mu \mathrm{m}$ in diameter we can determine the image's geometric center to better than $0.1 \mu \mathrm{m}$ for small Type-1 defects. Hence, from Fig. 7 , the sensitivity is $\pm 200 \AA$ in wall nonuniformities for a $S / N$ ratio of $1 .{ }^{12}$ To generalize this technique, curves similar to those shown in Fig. 7 need to be derived for variable wall thicknesses of 0.25 to $8.0 \mu \mathrm{m}$, for diameters of 50 to $800 \mathrm{\mu m}$, and for different material compositions. From these computerderived curies, an appropriate sensitivity curve can be extrapolated for a particular wall thickness, diameter, and material composition to analyze Type-I defects in most laser fusion targets.

B.

Type-II and -II Defect Sensitivities

The sensitivity of the T-test, or any other test, to detect wall-thickness variations for small Type-II and Type-III defects is determined by several factors: (1) by the mean optical density, D; (2) by the number, $N$, of independent density 
measurements; (3) by the readout system noise, ${ }_{0}$, derived from Fig. 3 and Eq. (1); (4) by the mean energy of the incident $x$ rays; (5) by the radial location of the defect from the center of the microsphere image, and (6) by the change in optical density per unit change in wall thickness, $d D / d l$. We have plotted $d D / d l$ versus photon energy in Fig. 8 for 1.5-jm-thick glass at various exposure levels on HRP emulsion. These factors result in an uncertainty in the wall thickness, $3 \sigma_{\ell}$, which limits the precision of the measurements of average glass thickness. The calculation of $30_{\ell}$ is defined below: 5,13

$$
3 o_{l}=3(d D / d \ell)^{-1} o_{D} N^{-1 / 2} \mu \mathrm{m} .
$$

For example, a 100-um-diam microsphere with a material thickness of $1.5 \mu \mathrm{m}(0.75-$ (um-thick wall) exposed to 3300 photons of CuL radiation per square micrometer results in $D=0.55, \sigma_{D}=0.043(F i g .3), d D / d \ell=0.3 \mathrm{um}^{-1}$ (Fig. 8), and, therefore, in $30_{\ell}=0.43 / \sqrt{N} \mu \mathrm{m}$. For our case, in which we step in $1-\mu \mathrm{m}$ increments, $N$ is related to the area scanned.

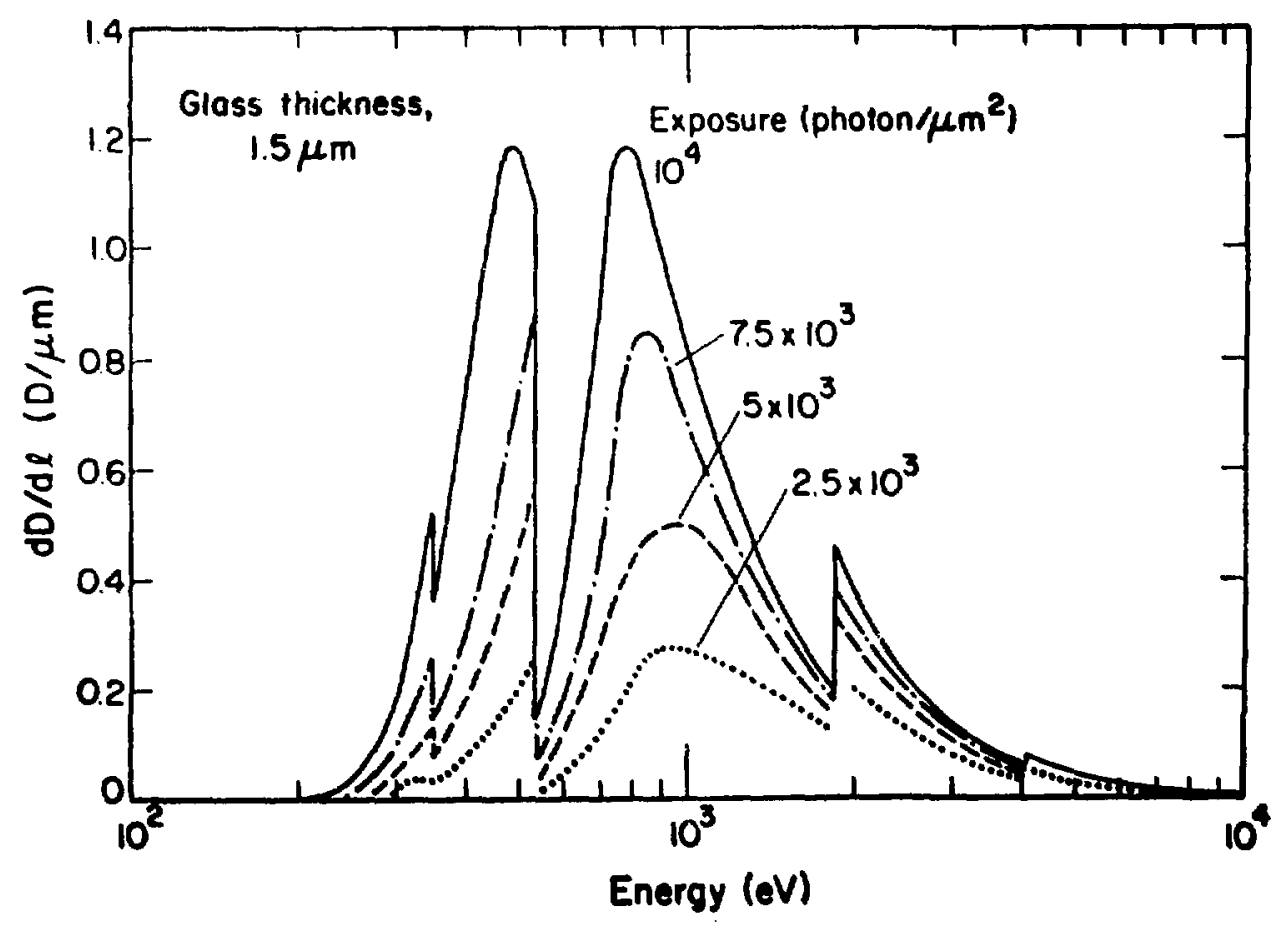

Fig. 8. Contrast as a function of energy. 
Cleven microspheres ware analyzed by using the calculation defined in Section IV. B for detecting defects with ratios of $S$ above 2 . We chose glass microspheres for this analysis to compare the sensitivities of the radiographic and optical interference techniques. The calculation was performed for 7-by 7-im areas (Fig. 6) from 10 to $95 \%$ of the microsphere's radius. The annulus over which each calculation was performed was stepped radially outward in 1-um steps. Note that the sampling aperture and the step size should be of the same size as the defect for which greatest sensitivity is required. These criteria will generate the greatest efficiency in total sampling time. Our calculation detected Type-ill defects violating the $S$ ratio of 3 , defined in Section IV. B. These defects ranged from $+300 \AA$ at $13 \%$ of the radius to $-1000 \AA$ at $85 \%$ of the radius.

These results allow a coarse comparison of the $+300-A$ defect in microsphere LBII (one of the defects for $S, 3$, Table 1), at $18 \%$ of the radius, with

\section{TABLE I}

NUMBER OF TYPE II AND III DEFECTS

Sample and
Exposure Date

LB1 $-7-15-77$

LB2 $-7-15-77$

LB3 $-7-15-77$

LBS $-7-15-77$

LB5 $-7-15-77$

LB6-7-15-77

LB7 $-7-15-77$

LB8 $-7-15-77$

LB $9-7-15-77$

LB 10-7-15-77

LB1]-10-13-77

\begin{tabular}{cc}
$2 \leq \frac{\text { Number of Defects }}{\mathrm{S}<3}$ & S 3 \\
\hline 6 & 1 \\
4 & 0 \\
0 & 0 \\
0 & 0 \\
4 & 0 \\
3 & $1 *$ \\
N/A & N/A \\
8 & 1 \\
1 & 0 \\
1 & 0 \\
19 & 3
\end{tabular}

* Excluding bad radiographic area. N/A Not available.

the $3 \sigma_{\ell}$ limit of Eq. (2). Microsphere LBII was approximately $335 \mu \mathrm{m}$ in diameter (Table II) with a wall thickness ranging between 0.76 and $1.24 \mu \mathrm{m}$ (Table III) depending on the measuring technique used. The microsphere was exposed to 
TABLE II

COMPARISON OF DIAMETER MEASUREMENTS

$$
\begin{aligned}
& \text { Sample and } \\
& \text { Exposure Date } \\
& \text { LB]-7-15-77 } \\
& \text { LB2-7-15-77 } \\
& \text { LB3-7-15-77 } \\
& \text { 1.84-7-15-77 } \\
& \text { LB5-7-15-77 } \\
& \text { LB6-7-15-77 } \\
& \text { LB7 }-7-15-77 \\
& \text { LB8 -7-15-77 } \\
& \text { LB9-7-15-77 } \\
& \text { LB) 1)-7-15-77 } \\
& \text { LB] }-10-13-77
\end{aligned}
$$

\begin{tabular}{|c|c|}
\hline $\begin{array}{l}\text { Sample Di } \\
\text { Two-Dimens iona } \\
\text { Analys is }\end{array}$ & $\begin{array}{l}\text { leter }(\mu \mathrm{m}) \\
\text { Interferometry }\end{array}$ \\
\hline 179 & .79 \\
\hline 183 & 184 \\
\hline 153 & 151 \\
\hline 181 & 184 \\
\hline 179 & 177 \\
\hline 186 & 185 \\
\hline 180 & 177 \\
\hline 180 & 179 \\
\hline 172 & 171 \\
\hline 176 & 174 \\
\hline 339 & 334 \\
\hline
\end{tabular}

TABLE III

COMPARISON OF WALL-THICKNESS MEASUREMENTS

Sample and

Exposure Date

LB] $-7-15-77$

LB2 $-7-15-77$

LB3 $-7-15-77$

LB4 $-7-15-77$

LB5 $-7-15-77$

LB6-7-15-77

LB $7-7-15-77$

LB8 $-7-15-77$

LB9-7-15-77

LB 10-7-15-77

LB11-10-13-77
Wall Thickness ( $\mu$ m) Two-Dimensional Analys is Interferometry Ratio

$\begin{array}{lll}1.14 & 1.37 & 1.20 \\ 1.11 & 1.44 & 1.30 \\ 0.72 & 0.84 & 1.17 \\ 0.98 & 1.56 & 1.59 \\ 1.00 & 1.19 & 1.19 \\ 1.00 & 1.23 & 1.23 \\ \mathrm{~N} / \mathrm{A} & 1.05 & \mathrm{~N} / \mathrm{A} \\ 0.98 & 1.21 & 1.23 \\ 1.03 & 1.28 & 1.24 \\ 1.11 & 1.55 & 1.40 \\ 1.24 & 0.76 & 0.61\end{array}$

N/A - Not available. 
- 8600 photons/i.m? of cul, radiation. This resulterd in a central density, 0, of

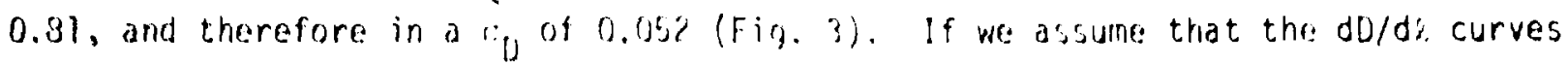
in Fig. B are approximately applicable for inicrosphere LEll, then an exposure of 8600 photons/im ${ }^{2}$ yields a do/di, of 1.0. These parameters substituted into [q. (2) result in $3 x_{0}=0.156 / \mathrm{N}$ im (good for the central ared only). The area used in the defect detection was $49, \mathrm{~m}^{2}$ and, therefore, $3 \%$, was about $\pm 225 \mathrm{~A}$. Comparison of the $+300-A$ detected defect with the $\pm 225-A$ detection limit implies that the two-dimensional radiographic image analysis technique can detect Tyle-III deferts very close to the theoretical limit, $3 ;$, in the central area.

The larger Type-II defects can be detected by using Ea. (2) and the technique of Appendix B, which converts the center optical density to thickness. If the center $4 \%$ of the microsphere, I.Bll, in the previous example was used to calculate a 30, , the value of $\mathrm{El}$. (2) would be $\pm 75 \mathrm{~A}$. Therefore, a Type-lI defect is detectable if several views have a central thickness that varies by more than $\pm 75 \AA$. An additional nethod for detecting large Type-II defects is a trend analysis of the rim radius used in Type-1 defect andysis.

We have outlined in preceding sections several techniques that can be used to determine three classes of defects. Quantitative results at present can be obtained only for glass microspheres over a limited range of wall thicknesses, diameters, and compositions. To increase the robustness of these techniques requires: (1) A complete set of $\mathrm{dD} / \mathrm{dl}$-vs-energy curves (Fig. 8) for various materials and thicknesses (e.g., 0.25 to $8.0 \mathrm{~lm})$; (2) a recalibration of the root mean-square (rms) film noise, o, versus film density (Fig. 3; this should be done periodically); (3) a set of sensitivity curves (Fig. 7) for targets of various diameters; and (4) a step tablet calibration for HRP emulsion over an optical density range of 0.0 to 3.0 on the PDS microdensitometer.

To analyze all three classes of defects, we have implemented algorithms on our CDC 7600. For an original 128-by-128, 8-bit gray-level laser fusion target image, the processing time is about 8 to $10 \mathrm{~s}$. For 256-by-256 and 512-by-512 images, the processing time is 40 and $160 \mathrm{~s}$, respectively.

VI. COMPARISON OF EXPERIMENTAL RESULTS

We will now describe the quantitative results of the techniques we have described and will compare the results of some of these techniques with those of other image-analysis systems. These systems are: (1) two-dimensional computer analysis using digitized radiographic data from a PDS microdensitometer, (2) an 
analog video system from Interpretation Systems, Inc, with a large-magnification microscope for input of the radiographic data, and (3) a Jamin-Lebedev optical i: terferometric measuring system, also using a large-magnification microscope for input. 'iur data base is a set of eleven microspheres that were radiographed with Cul radiation.

We will describe: (1) the size of Type-I defects that can be detected with the three systems, (2) the results of identifying Type-II and Type-III defects using the statistical outlier techniques, and (3) the optical interferometric measurements of diameter and central thickness of the radiographed microspheres made with the two-dimensional computer analys is technique.

A.

Type-I Defect Comparison

To compare the results of the three analysis techniques for measuring the size of Type-I defects, we have computed two ratios: (1) the ratio of eccentricity measured with interferometry (as listed ir Table IV) vs eccentricity measured with

TABLE IV

SIZES OF TYPE -I DEFECTS MEASIJRED THREE WAYS

Sample and

Exposure Date

LB] $-7-15-77$

LB2 $-7-15-77$

LB3-7-15-77

LB4 $-7-15-77$

LB5 $-7-15-77$

LB6 $-7-15-77$

LB7 $-7-15-77$

L.B8-7-15-77

LB9-7-15-77

LB10-7-15-77

LB11-10-13-77
Eccentricities Determined by Various Methods (\%) Two-Dimensional Optical Computer* Interferometry 3

$11(18+)$

10

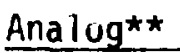

4

5

$15(21+)$

12

5

6

N/A

$\leqslant 2$

11

5

5
8

3

9

6

$21+$

13

5

8

$5 \quad 5$

$10 \quad 10$

56

$3<5$
$4<5$

* Based on experimental curves for 80- $\mu m$-diam laser fusion target with 1-um-thick wall.

* Minimum sensitivity of this method is $\pm 5 \%$.

+ These eccentricities were found at $1 / 4$ of the radius.

N/A Not available. 
the two-dimensional computer technique, and (2) the ratio of eccentricity measured with optical interferometry vs eccentricity measured with the analog-image andlyzer. These results are plotted in Fig. 9. Only Type-1 defects in excess of $\pm 500 \wedge$ were considered because this size is approximately the limit of sensitivity of both the analog-image analyzer and the optical interferometric measurements. However, there are phase-sensitive interferometry techniques that have been shown to be sensitive to $\pm 100 \AA$. 14,15

For the two-dimensional computer analysis technique the average ratio is $0.99 \pm 0.37$, whereas for the analog-image analyzer the ratio is $1.02 \pm 0.30$. Thus, the two-dimensional computer analys is technique detects eccentricities as small as those detected by the best limit sensitivity of the interferometric and analog-image-analyzer techniques.

While optical interferametry provides a means of quick inspection of optically transparent targets, the two-dimensional analysis of radiographic images is a sensitive means of measurirg defect sizes even in opticaliy opaque riaterials. B. ....... Type-1i and -1 II Defect Results

As discussed in Section V.B, smaller Type-II and Type-III defects were detected with an outlier technique using the statistical T-test. The total number of defects with S-ratios between 2 and 3, and above 3, are listed in Table 1 for each of the 11 microspheres. Figure 10 indicates by square boxes the positions

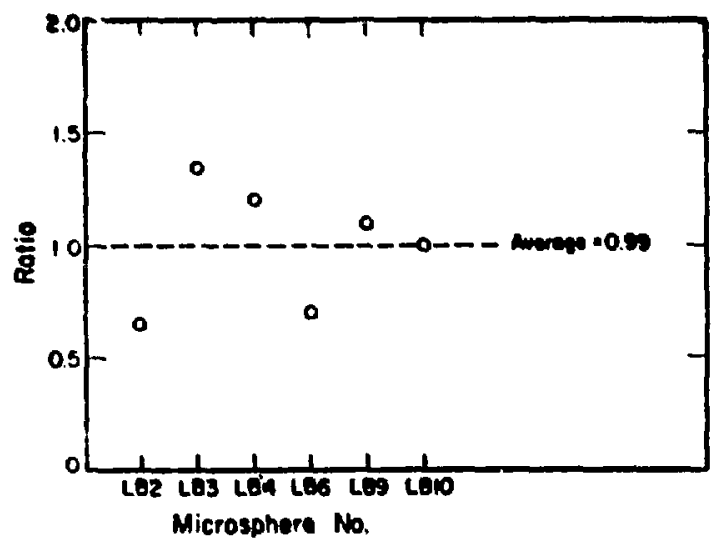

Fig. 9a. Type-I defect ratios for twodimensional analysis.

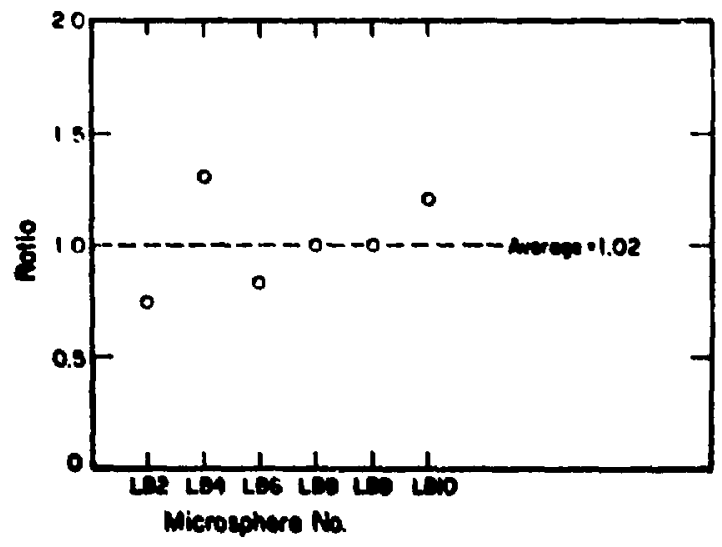

Fig. 9b. Type-I defect ratios for analog-image analyzer. 


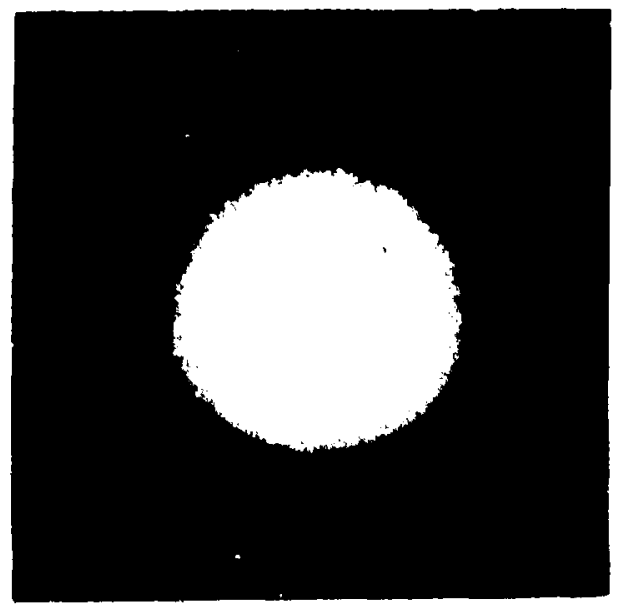

Fig. 10. Laser fusion target imare with eight Type-III defects outlined.

of eight of the 22 defects with an S-ratic greater than 2 for microsphere LB?l in Table I. The innermost defect in Fig. 10 has an S-ratio corresponding to a defect size of $+300 \mathrm{~A}$, which cannot be seen by the unaided eye in the photograph. The three outermost defects on the left side of Fig. 10 have S-ratios between 2 and 3 , which correspond to thickness variations between -1000 and $-2000 \AA$. C. Diameter and Wall-Thickness Comparison

The 11 computer-calculated and interferometrically measured microsphere diameters are shown in Table II. The diameters measured compare closely for all microspheres. Some of the small variations may be due to: (1) PDS edge response to large density changes, (2) the convolution of the 2-um aperture of the PDS with the 1- $\mu \mathrm{m}$ wall thickness, (3) x-ray edge diffraction, (4) HRP film shrinkage characteristics, and (5) distance calibration in the two devices.

A comparison of the wail thickresses in Table III shows that a wall thickness measured interferometrically is $1.28 \pm 0.13$ times that measured from the radiographic image. This systematic difference is caused by: (1) small changes in the index of refraction of the target due to composition variations; (2) small changes in the $x$-ray absorption coefficient due to composition variations; (3) uncertainty in the $\mathrm{H}-\mathrm{D}$ curve of the film; and (4) uncertainty in total exposure. The first two of these problems can be minimized by detailed spectroscopic analysis on each batch of targets or by $x$-ray fluorescence analys is of the targets during radiography. Problems 3 and 4 , as well as problem 2, can be controlled by 
carefully calibrating each exposure with a step tablet of known step size made of the same material as the shells.

Careful measurement of the total wall thickness and target diameter is required to quantify the size of various defects. For Type-I defects it is necessary to know the total wall thickness and target diameter before one can construct curves as those shown in Fig. 7 to determine the defect size. Similarly, Type-II and -III defects are dependent on the precision of Fig. 2 and the $x$-ray absorption coefficient. When optically opaque laser Fusion targets or opaque material layers are used, we will have to rely totally on these analytical procedures.

\section{IMPROVED IMAGE REAUOUT SYSTEMS}

The sensitivity 1 imit of $x$-ray micruradiography to different types of defects is more than adequate for present needs and should be acceptable for future applications. However, to improve the time efficiency of the system, we foresee the characterization of future laser targets as comprising a multistep process with each stop representing a refinement in the sensitivity to defects. A trade-off can then be made between the level of acceptable nonuniformity and the manpower and computational effort expended to characterize the microspheres. The first step would be visual inspection of the radiographic images for symmetry, gross wall-thickness nonuniformities, and surface defects. Acceptable targets would then be radiographed again in three orientations and the images scanned with an analog-image analyzer to eliminate targets with nonuniformities greater than $10 \%$. The inages of the remaining targets would then be analyzed by computer. Since many microspheres can be radiographed simultaneously, the initial steps should represent only a small fraction of the total time needed to characterize a microsphere.

Three methods of scanning densitometry for the computer analysis were initially investigated to minimize the total analysis time; they were: 1) flying spot scanner, 2) camera scanner, and 3) densitometer scanner with an $x$-and $y$-stage. Based on parameters outlined in Appendix $C$ for each scanning system, the flying spot scanner has the best performance. This is a result of: 1) minimal $z$-axis shading correction and $x$ - and $y$-geometric distortion correction unlike the vidicon camera system, and 2) not having to mechanically step an $x$ - and $y$-stage as in the densitometer system. As a result a careful look at the CrDAC flying spot scanning system at LLL was made. Its main drawback was that it was expensive to buiid. A state-of-the-art CCD camera system looks like the best compromise at this time. 
It achieves high data rate at relatively low cost while sacrificing analys is time to make the necessary corrections to the data. However, whichever scanning system is chosen, maximum utility will be achieved by digitizing the data and interfacing this readout system directly to a dedicated computer to maximize throughput.

\section{VII:. CONCLUSIONS}

The most important feature of x-ray microradiography combined with twodimensional computer analysis is the high level of sensitivity to spherical and local wall-thickness variations in opaque laser fusion targets. In addition, the sensitivity of microradiography for optically transparent laser fusion targets is slightly better than that of most optical interferometric techniques; however, recently developed phase-sensitive interferometers are more sensitive to wallthickness variations.

The sensitivity of $x$-ray microradiography can be improved by more detailed scans of the image, by projection radiography, or by averaging the results of many successive scans to reduce noise. A more complete result for Type-III defects would include a determination of the defect. mass by first determining the area of the defect using feature-clustering methods. For more robust Type-I defect analysis, the wall-thickness and diameter dependencies of curves similar to those of Fig. 7 need to be derived. In addition, Moiré pattern analysis, two-dimensional Fourier analysis, and $\rho-\theta$ transformations may provide a higher sensitivity in some or all classes of defects.

\section{ACKNOWLEDGMENTS}

We gratefully acknowledge the aid of the following LASL colleagues: R. Bagley for performing the scanning densitometry, B. Carpenter for film processing, and $M$. Campbell for devising and implementing numerous clever ways to mount laser fusion targets. We thank P. Lyons of LASL, P. Bartels of the University of Arizona, and H. C. Andrews of the University of Southern California, for many useful discussions concerning all aspects of the work reported here. 


\section{APPENDIX A}

TWO-DIMENSIONAL MODELING

The geometry of the model used in generating the simulated microspheres is shown schematically in Fig. A-1. A spherical shell with outer surface $x^{2}+y^{2}+z^{2}=R 0$ and inner radius $R 1$ has the center of the inner wall displaced by Ex and Ey from the center of the outer wall in the $x$-and $y$-directions, respectively. The $x$-ray source is modeled as a circular aperture of radius $r_{a}$ orthogonal to the $z$-axis at $z=z_{1}$. The source is subdivided into 100 equal-area regions. The plane $z=-R O$ defines the film plane on which we establish a $1-\mu m$

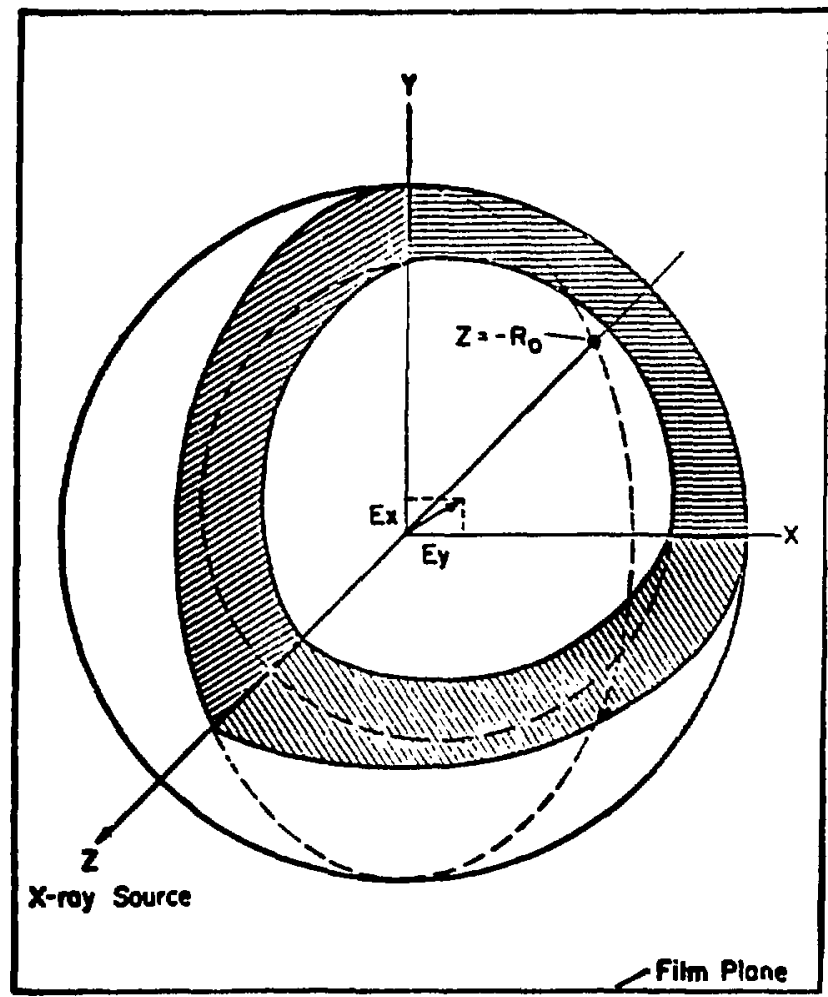

Fig. A-1. Two-dimensional analys is geometry. 
square grid of pixels (image or picture elements) sufficient to cover the image. Each grid point is subdivided into a 0.1 - by $0.1-\mu \mathrm{m}$ subgrid, and the transmission through the target $t(k, l)$ is computed at each point on the subgrid as the average transmission for each of the 100 source points.

The transmission is derived by calculating the length of target material between each subgrid point and each source point and then multiplying this thickness, $d(k, l, m, n$,$) , by the known x$-ray absorption coefficient, $u$, for the target material:

$$
t(k, l)=\sum_{m, n=1}^{10} e^{-u x d(k, l, m, n)} .
$$

The transmissions at each subgrid point are then averaged to generate the average transmission $T(i, j)$ for each pixel.

For an initial photon flux, $H_{0}$, the mean optical density in each pixel can be determined by calculating the flux in each pixel $\left(H(i, j)=H_{0} \cdot T(i, j)\right)$, and referring to Fig. 2 of the body of the report, converting photon flux to optical density. This procedure creates the simulated radiographic target images upon which the sensitivity analys is is performed.

\section{APFENDIX B}

RELATION OF MEAN OPTICAL DENSITY TO MATERIAL THICKNESS

The procedure for converting the mean optical density to thickness in micrometers for a specific material involves the following steps.

(1) Determine the $x$-ray exposure $H$ for a given optical density, $D$, from the $H-D$ curve (Fig, 2).

(2) Determine the $x$-ray exposure $H_{0}$ that corresponds to the background density $D_{0}$.

(3) Calculate the $x$-ray absorption coefficient, $\mu(E)$, of the material used from the absorption coefficients of Henke ${ }^{16}$ (Fig. B-1).

(4) The thickness, T, of the material is given by

$$
T=\frac{1}{u(E)} \ln \left(H / H_{0}\right) .
$$




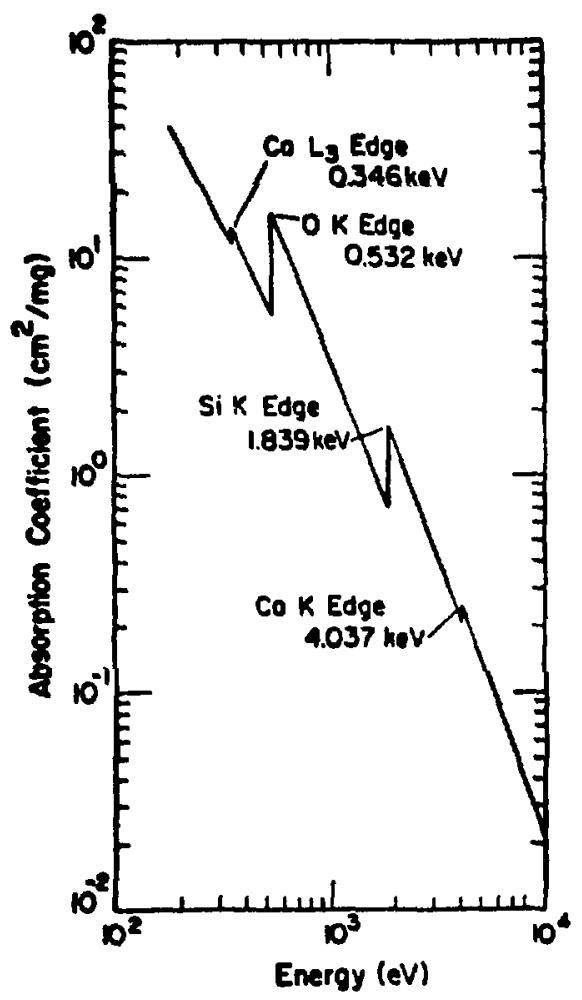

Fig. B-1. X-ray absorption coefficient vs energy for a typical batch of microspheres.

This procedure can be reversed to obtain optical density, as was done in Sections III and IV in which computer-simulated laser fusion targets were computed and analyzed.

\section{APPENDIX $C$}

\section{THREE SCANNING SYSTEM PARAMETERS}

A list of the important parameters about each scanning system follows.

1. Flying spot scanner
a) spatial resolution $\sim 0.3 \mu \mathrm{m}$
b) z-axis shading - $0.1 \%$
c) $x$ - and $y$-geometric distortion - $0.1 \%$
d) scanning speed $>10 \mathrm{Mhz}$
e) field of view - 700 micrometers
f) adjacency effects - small 
g) afterglow - N/A

*h) two density units of discrimination - limited by microscope optics

2. Densitometer scanner with $x$ - and $y$-Zeiss stage
a) spatial resolution $\sim 0.5 \mu \mathrm{m}$
b) z-axis shading - with care $1 \%$
c) $x$ - and y-geometric distortion - with care $1 / 2 \%$ to $1 \%$
d) scanning speed - $10 \mathrm{kHz}$ (Zeiss $x$ - and $y$-stage)
e) field of view - 1 inch by 3 inches (Zeiss $x$ - and $y$-stage)
f) adjacency effects - small
g) afterglow - N/A
*h) two density units of discrimination - limited by microscope optics

3. Vidicon camera scanner**
a) spatial resolution $\sim 0.5 \mu \mathrm{m}$
b) z-axis shading correction 1\% (IMANCO)
c) $x$ - and $y$-geometric distortion correction $\sim 1 / 2 \%$ over $80 \%$ of the field of view
d) scarning speed $\sim 4 \mathrm{Mhz}$
e) field of view - 700 micrometers with special lens
f) adjacency effects - small (for types other than S-T vidicon it could be sizable)
g) afterglow - for a dynamic image or constantly changing field of view afterglow is a problem

*h) two density units of discrimination

* When interpreting parameter $h$ of each scanning system it should be noted that eight or nine bits over the two density units is possible with multiple frame averaging.

** Silicon vidicon is the most advantageous choice of camera scanners for this problem. 


\section{REFERENCES}

1. B. W. Weinstein, J. App. Phys, 46, 5305 (1975).

2. B. W. Weinstein, C. D. Hendricks, "Interferometric Measurement of Laser Fusion Targets," Lawrence Livermore Laboratory Report UCRL-78477 Rev. 1.

3. T. M. Henderson, D. E. Cielaszyk, and R. J. Simms, Rev. Sci. Instrurn. 48, 835 (1977).

4. R. H. Day and E.J.T. Burns, Adv. X-ray Anal. 19, 597 (1976).

5. R. H. Day, T. L. Elsberry, R. P. Kruger, D. M. Stupin, and R. L. Whitman, "X-ray Microradiography of Laser Fusion Targets," Proc. Of the Eighth Int. Conf. on X-ray Optics and Microanalysis, Boston, Massachusetts, August 1977.

6. B. L. Henke, Tech. Rep. No, 4, AFOSR Contract No. AF 49(638)-394, unpubl ished (1961).

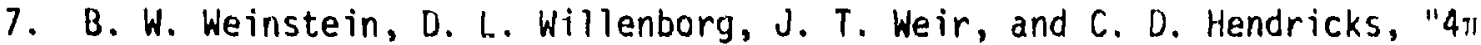
Interferometric Measurements of Laser Fusion Targets," Proc. of Topical Meeting on Inertial Confinement Fusion, San Diego, California, February 7-9, 1978, Opt. Soc. of Amer., 78CH1370-ZQEA, p. TuE9-1.

8. H. C. Andrews and B. R. Hunt, Digital Image Restoration (Prentice-Hal), New York, 1977), pp 20-23.

9. R. E. Moore, Mathematical Elements of Scientific Computing (Holt, Reinhart and Winston, New York, 1975).

10. D. M. Himmelblau, Applied Nonlinear Programming (McGraw-Hill, New York, 1972).

11. Frank E. Grubbs, "Procedures for Detecting Outlying Observations in Samples," Technometrics 11, No. 1, pp 1-2, February 1969.

12. R. L. Whitman, R. P. Kruger, R. H. Day, and D. M. Stupin, "Two-Dimensional Computer Modeling and Analys is of Thin-Wall Microspheres," Proc. of the Topical Meeting on Inertial Confinement Fusion, San Diego, California, February 7-9, 1978, Opt. Soc. of Amer., 78CH1310-ZQEA, p. TuE13-1.

13. D. M. Stupin, R. H. Day, R. L. Whitman, and R. P. Kruger, "Microradiography of Laser Fusion Targets with Monochromatic X-rays," ibid, p. TuE12-3.

14. P. 0. McLaughl in and D. T. Moore, "Laser Fusion Microballoon Characterization Using a Single-Pass AC Interference Microscope," Abstract No. TUQ16, Meeting of the Opt. Soc. of Amer., Toronto, Canada, October 1977. (Gradient Index Lab. Report. No. GI0-10, The Institute of Optics, University of Rochester, Rochester, New York). 
15. G. W. Johnson and D. T. Moore, "Design and Construction of a Phase-Locked Interference Microscope," The Soc. of Photo-Optical Instrumentation Engineers, 103, Systems Integration and Optical Design II, 76 (1977).

16. B. L. Henke and E. S. Ebisu, Adv. X-ray Anal, 1\%, 150 (1973). 